University of Nebraska - Lincoln

DigitalCommons@University of Nebraska - Lincoln

4-1-1996

\title{
Analysis of homogeneous combustion in Monolithic structures
}

Hendrik J. Viljoen

University of Nebraska - Lincoln, hviljoen1@unl.edu

Follow this and additional works at: https://digitalcommons.unl.edu/chemengchemreact

Part of the Chemical Engineering Commons

Viljoen, Hendrik J., "Analysis of homogeneous combustion in Monolithic structures" (1996). Papers in Chemical Reactions. 2.

https://digitalcommons.unl.edu/chemengchemreact/2

This Article is brought to you for free and open access by the Chemical and Biomolecular Engineering Research and Publications at DigitalCommons@University of Nebraska - Lincoln. It has been accepted for inclusion in Papers in Chemical Reactions by an authorized administrator of DigitalCommons@University of Nebraska - Lincoln. 


\title{
ANALYSIS OF HOMOGENEOUS COMBUSTION \\ N MONOLITHIC STRUCTURES
}

\begin{abstract}
An analytical method is used to solve the problem of homogeneous combustion in a monolith. The solid phase acts as burner support. Heat is conducted much more effectively in the solid phase and preheating of the gas is more effective. The flame temperature exceeds the adiabatic value. Solutions are presented for different velocities and feed concentrations.
\end{abstract}

\section{INTRODUCTION}

Both catalytic and gas-phase combustion reactions can be carried out in the presence of a solid medium. Examples of the first type include catalytic oxidation reactions, where the support can be pellets or a monolithic structure (Heck et al., 1976; Oh et al., 1993; Papenmeier and Rossin, 1994). Gas-phase combustion reactions in solid media find application as burners. Porous radiant burner supports consist of reticulated alumina, spongelike metal or honeycomb monoliths (Min and Shin, 1991; Sathe et $a / ., 1990$ ).

The phenomenon of super adiabatic combustion was first described by Weinberg in 1971; he referred to it as excess enthalpy combustion. Premixed combustion in porous media results in flame temperatures above the adiabatic value due to the preheating of the inlet stream by the solid phase (Min and Shin, 1991). Although the extent of the thermal overshoot is enhanced by radiative feedback and reciprocal feedstreams, it also occurs in a unidirectional feed configuration where radiative heat transfer is neglected.

Thermal overshoots in the gas phase compromise the system in several ways, and we will briefly discuss two effects. The high gas temperature can alter chemical equilibrium. For example, it is expected that the combustion of a lean methane-air mixture will produce combustion products $\mathrm{CO}$, and $\mathbf{H 2 0}$ only. At temperatures above $1900 \mathrm{~K}$, however, CO starts to form (along with traces of $\mathrm{NO}$ ) and the equilibrium rapidly shifts toward $\mathrm{CO}$ with a further increase in temperature. The overshoots can also affect the mechanical integrity of the monolith. In non-adiabatic burner systems a considerable amount of heat is radiated from the solid phase toward the load. If the system is designed to operate at a maximum temperature less than the adiabatic flame temperature, this maximum temperature can be exceeded. Thus, thermal overshoots as a result of super adiabatic combustion may lead to equilibrium shifts and also chemical or mechanical failures of the solid medium (Chen et al., 1992; Sih and Ogawa, 1982; Thiart et al., 1991).

The purpose of this note is to demonstrate an analytical method to study combustion in a two phase system. The flame front position and the temperature overshoot as a function of the feed velocity are also investigated.

Comment: This paper was originally published in the Journal of "Chemical Engineering Science", Vol.51, No.7, pp.1107-1111, 1996 (C) of this paper belong to Elsevier Science Ltd. 


\section{MODEL}

Consider a single monolith channel of finite length $\mathrm{L}$ in an adiabatic system. Diffusion effects and radial conduction are neglected and the thermophysical properties are assumed constant. The pressure drop in the monolith is neglected and an average pressure is used in the equation of state. We have to make these assumptions to keep the analysis tractable. The governing equations are:

$$
\begin{aligned}
& \rho_{f} C_{p_{f}} \frac{\partial T_{f}}{\partial t}= k_{f} \frac{\partial^{2} T_{f}}{\partial x^{2}}-\rho_{f} C_{p_{f}} U \frac{\partial T_{f}}{\partial x} \\
&-\frac{4 h_{w}}{D}\left(T_{f}-T_{w} !\right. \\
&+(-\Delta H) C k_{0} \exp \left(\frac{E}{R_{g} T_{f}}\right) \\
& \rho_{w} C_{p_{v}} \frac{\partial T_{w}}{\partial t}=k_{w} \frac{\partial^{2} T_{w}}{\partial x^{2}}-\frac{h_{w}}{\Delta d}\left(T_{f}-T_{w}\right) \\
& \text { 鲌 }=-\frac{\partial U C}{\partial x}+C k_{0} \exp \left(-\frac{E}{R_{g} T_{f}}\right) \\
& \rho=\frac{P}{R_{g} T_{f}} .
\end{aligned}
$$

The maximum flame temperature is $\mathrm{T}$, (usually more than the adiabatic value $\mathrm{T}$,). Using the length of the monolith as the length scale, L/Uo for the timescale, Co for concentration and (T, - To) for temperature, the following dimensionless variables are defined: $\chi=x / L, \tau=(U o, / L) t, \quad \zeta=C / C o, \theta=(T-T o) /(T,-T o), p=U / U o$. Suppose the flame is stabilized at a distance $f o$ from the inlet. The axial coordinate is transformed such that the origin is at the flame front, i.e. situated at zero, by $\quad \mathrm{z}=\boldsymbol{x}-1 ;=0$. The steady state combustion model is described by the following dimensionless equations:

$$
\begin{gathered}
0=\frac{1}{P e_{f}} \frac{\partial^{2} \theta_{f}}{\partial z^{2}}+\phi_{1}\left(\theta_{f}-\theta_{w}\right)+\beta D a \zeta \exp \left[\frac{\gamma\left(\theta_{f}-1\right)}{\theta_{f}+\sigma}\right] \\
0=\frac{1}{P e_{w}} \frac{\partial^{2} \theta_{w^{\prime}}}{\partial z^{2}}+\phi_{2}\left(\theta_{f}-\theta_{w}\right) \\
0=-\frac{\partial \mu \zeta}{\partial z}-D a \zeta \exp \left[\frac{\gamma\left(\theta_{f}-1\right)}{\theta_{f}+\sigma}\right]
\end{gathered}
$$

That $\beta$, Da and $\gamma$ are not known apriori for this formulation, since they are defined in terms of $\mathrm{T}$,. The boundary conditions are: 


$$
\begin{gathered}
\frac{\partial \theta_{w}}{\partial z}=0, \quad z=-f_{0} \\
\theta_{f}=\frac{1}{P_{\ell_{f}}} \frac{\partial \theta_{f}}{\partial z}, \quad \bar{z}=-f_{0} \\
\zeta=1, \quad z=-f_{0} \\
\frac{\partial \theta_{x^{\prime}}}{\partial z}=0, \quad z=1-f_{0} \\
\frac{\partial \theta_{f}}{\partial z}=0, \quad z=1-f_{0} .
\end{gathered}
$$

\subsection{ANALYSIS OF REACTION ZONE.}

The large activation energy of the combustion reaction limits the chemical reaction to a thin zone. This is the so-called flame sheet approximation (Matkowsky and Sivashinsky, 1979: Norbury and Stuart, 1989). Most analyses of this kind have been done on an infinite domain, i.e. $\mathbf{x} \dot{E} \mathbf{R} 1$. This analysis differs from previous work in that the domain of the solution is finite,. $\mathbf{x} \mathrm{E}[0, \mathrm{~L}]$. In the analysis on an infinite domain, the flame velocity was considered an unknown. Steady-state combustion is then associated with a constant speed of the combustion front. In the case of a finite analysis, the flame position within the monolith is not known a priori. An asymptotic analysis of the flame front provides us with an analytical solution of the model. It also provides us with the necessary information to find the axial flame position fo The analysis is done by matching the solutions inside the combustion front with the solutions outside the combustion front. Inside the combustion front the axial variable is stretched as $v=y z$ and the following expansions are made:

$$
\begin{gathered}
\theta_{r}=\theta_{r 0}+\frac{1}{\gamma} \theta_{r 1}+\ldots \\
\zeta=\zeta_{0}+\frac{1}{\gamma} \zeta_{1}+\cdots \\
\mu=\mu_{0}+\frac{1}{\gamma} \mu_{1}+\cdots \\
D a=\gamma D a_{0}+D a_{1}+\frac{1}{\gamma} D a_{2}+\cdots
\end{gathered}
$$

Collecting terms of $O(1)$,

$$
\frac{\mathrm{d}^{2} \theta_{r 0}}{\mathrm{~d} v^{2}}=0
$$

And matching the solution with the outer solutions $\boldsymbol{u}$ results in $\mathrm{H},,=1$. Collecting terms of $\mathrm{O}$ $(1 / \gamma)$, we get 


$$
\begin{gathered}
\frac{1}{P e_{s}} \frac{\mathrm{d}^{2} \theta_{r 1}}{\mathrm{dv}^{2}}+\beta D a_{0} \zeta_{0} \exp \left(\frac{\theta_{r 1}}{1 \neq \xi}\right)=0 \\
\mathrm{~d} \mu_{0} \zeta_{0}=D a_{0} \zeta_{0} \exp \left(\frac{\theta_{r 1}}{1+\sigma}\right) \\
\mathrm{d} v
\end{gathered}
$$

As $\mathrm{v} \rightarrow+\infty$, the fuel is completely consumed and $\zeta_{\mathrm{o}}(+\infty, t)=0$. In the same limit, $\mathrm{d} \theta_{\mathrm{r} 1} / \mathrm{d} v$ must match the outer solution expressed in terms of $v$. Let this limiting value be denoted as $\alpha+$. Combining eqs (18) and (19) and integrating between $v$ and $+\infty$ we

find a relationship between $\zeta_{0}, \mu_{0}$ and $\mathrm{d} \theta_{r 1} / \mathrm{d} v$ :

$$
-\beta \mu_{0} \zeta_{0}=\frac{1}{P e_{f}}\left(\alpha^{+}-\frac{\mathrm{d} \theta_{r 1}}{\mathrm{~d} v}\right)
$$

It follows from the dimensionless form of eq. (4) that

$$
\mu_{0}=1+\frac{1}{\sigma}
$$

Combining eqs (20) and (21) results in

$$
\zeta_{0}=\frac{1}{P e_{r} \beta} \frac{\sigma}{\sigma+1}\left(\alpha^{+}-\frac{\mathrm{d} \theta_{r 1}}{\mathrm{~d} v}\right) \text {. }
$$

$\mathrm{v}$ is bounded from below as $-\gamma f o$. We consider $\mathrm{y}$ to be large but finite. As $\mathrm{v} \rightarrow-\gamma f o, \mathrm{~d} \theta_{\mathrm{r} 1} / \mathrm{dv}$ approaches the stream up value of $\boldsymbol{\alpha}^{-}$and $\zeta_{0}=\mu_{0}=1$. From eq. (20) it is seen that

$$
\alpha^{-}-\alpha^{+}=P e_{f} \beta
$$

Substituting eq. (22) into eq. (18) and integrating between $-\gamma f o$ and 0 , we find

$$
\begin{aligned}
- & \alpha^{-}+\alpha^{+} \ln \left(\frac{\alpha^{+}}{\alpha^{+}-\alpha^{-}}\right) \\
& =-D a_{0} \sigma\left\{1-\exp \left[\frac{-\gamma f_{0}}{(1+\sigma)}\right]\right\} .
\end{aligned}
$$

The matching values of $\boldsymbol{\alpha}^{-}$and $\boldsymbol{\alpha}^{+}$are given by the solutions outside the reaction zone. Equation (24) gives a relationship between the outer solutions $\left(\boldsymbol{\alpha}^{-}\right.$and $\left.\boldsymbol{\alpha}^{+}\right)$and the flame's position. 


\subsection{SOLUTION OF THE EQUATIONS}

The flame sheet approximation allows the problem to be viewed in three zones, i.e. the precombustion zone. The combustion zone and the post-combustion zone. For the precombustion zone the temperature can be written as

$$
\theta_{f}^{-}=A e^{\lambda_{1} x}+B e^{\lambda_{2} x}+C e^{\lambda_{3} z}
$$

Likewise, the temperature for the post-combustion zone is

$$
\theta_{f}^{+}=D e^{\lambda_{1} z}+E e^{\lambda_{2} z}+F e^{\lambda_{3} z} .
$$

Note that $\alpha^{-}$and $\alpha^{+}$are given by

$$
\begin{aligned}
& \alpha^{-}=\lambda_{1} A+\lambda_{2} B+\lambda_{3} C \\
& \alpha^{+}=\lambda_{1} D+\lambda_{2} E+\lambda_{3} F .
\end{aligned}
$$

The coefficients A -F, $T_{m}$ and flame position fo can be solved using eqs (9), (11), (24) and the following conditions at the combustion zone $\left([]=.[.]^{+}-[.]^{-}\right)$:

$$
\begin{gathered}
{\left[\theta_{f}\right]=0} \\
{\left[\theta_{w}\right]=0} \\
{\left[\frac{\mathrm{d} \theta_{w}}{\mathrm{~d} x}\right]=0} \\
{\left[\frac{\mathrm{d} \theta_{f}}{\mathrm{~d} x}\right]+P e_{f} \beta=0 .}
\end{gathered}
$$

The system is closed by

$$
\theta_{r}=1, \quad z=0 .
$$

Table 1 shows the properties for methane combustion (Sathr et al., 1990). The large $\mathrm{Pe}_{\mathrm{f}}$ and $P e_{w}$, numbers cause difficulty in the actual solution of the equation. This problem was overcome by the appropriate rescaling of the coefficients $\boldsymbol{A}$ and $\mathbf{D}$. (See Table 2 for parameter values for a methane content of $6 \%$ and a velocity of $0.5 \mathrm{~m} / \mathrm{s}$.]

\section{RESULTS AND DISCUSSION}

The flame position corresponding to a certain maximum temperature is determined for methane fractions of 4, 6 and $8 \%$. This is done for a series of feed velocities. Figure 1 shows the solution found near the inlet, and Fig. 2 shows the solution found near the outlet of the monolith. For each fraction a minimum velocity exists, below which no solution is found. In order to sustain the combustion reaction, a minimum fuel flux is required and the flame extinguishes if the velocity drops below the minimum associated with this flux. This minimum velocity increases when the fuel fraction of the feed is increased. At $4 \%$ the lower limit on the velocity is $0.02 \mathrm{~m} / \mathrm{s}$ and at $6 \%$ it is $0.12 \mathrm{~m} / \mathrm{s}$. 
A limit also exists for the maximum velocity. The solutions at the inlet and at the outlet approach the limit as the velocity is increased. This limit is associated with a limit point of the bifurcation diagram which can be constructed by plotting the flame position as a function of velocity (i.e. Figs 1 and 2). The blow-off velocities vary from $0.33 \mathrm{n} \sim / \mathrm{sat} 4 \%$ to $1.28 \mathrm{~m} / \mathrm{s}$ at $8 \%$.

In Fig. 3 the maximum temperature of the fluid phase is plotted as a function of the velocity for a flame stabilized near the inlet. The maximum temperature increases as velocity is increased. At first glance this trend may appear counterintuitive, but it must be kept in mind that the controlling mechanism of this reaction is mass transfer and the molar flux of fuel increases when the velocity is increased. Near the limit (blow-off velocity) the gas temperature exceeds the adiabatic temperature by a considerable amount. This overshoot decreases when the methane fraction is increased. For a feed of $4 \%$ methane the adiabatic

Table 1. Properties for methane combustion

\begin{tabular}{ccc}
\hline Item & Units & Value \\
\hline$C_{p_{x}}$ & $\frac{\mathrm{J}}{\mathrm{mol} \mathrm{K}}$ & 40 \\
$C_{p_{w}}$ & $\frac{\mathrm{J}}{\mathrm{kg} \mathrm{K}}$ & 1225 \\
$D$ & $\mathrm{~m}$ & 0.004 \\
$\Delta d$ & $\mathrm{~m}$ & 0.0015 \\
$E$ & $\frac{\mathrm{J}}{\mathrm{mol}}$ & 130,000 \\
$\Delta H$ & $\mathrm{~J}$ & $8 \mathrm{n} 10^{5}$ \\
$h_{w}$ & $\frac{\mathrm{Wol}}{\mathrm{m}^{2} \mathrm{~K}}$ & 137.3 \\
$k_{f}$ & $-\frac{\mathrm{W}}{\mathrm{m} \mathrm{K}}$ & 0.15 \\
$k_{0}$ & $\frac{1}{\mathrm{~s}}$ & $1.8 \times 10^{8}$ \\
$k_{w}$ & $\frac{\mathrm{W}}{\mathrm{m} \mathrm{K}}$ & 27 \\
$L$ & $\frac{\mathrm{m}}{\mathrm{J}}$ & 0.1 \\
$R_{g}$ & $\frac{\mathrm{mol} \mathrm{K}}{\mathrm{kg}}$ & 8.314 \\
$\rho_{s}$ & $\frac{P M}{\mathrm{~m}^{3}}$ & $R_{g} T$ \\
$\rho_{w}$ & $\frac{\mathrm{kg}}{\mathrm{m}^{3}}$ & 2500 \\
\hline & & \\
\hline & &
\end{tabular}


Table 2. Parameter values - $6 \%$ methane, $\mathrm{U}=0.5 \mathrm{~m} / \mathrm{s}$

\begin{tabular}{cc}
\hline Parameter & Value \\
\hline$D a$ & 15,576 \\
$P e_{s}$ & 541.6 \\
$P e_{w}$ & 5671 \\
$\beta$ & 0.7 \\
$\gamma$ & 7.7 \\
$T_{u}$ & $1504 \mathrm{~K}$ \\
$T_{m}$ & $2018 \mathrm{~K}$ \\
$T_{0}$ & $300 \mathrm{~K}$ \\
$\phi_{1}$ & 16.9 \\
$\phi_{2}$ & $5.98 \times 10^{-3}$ \\
\hline
\end{tabular}

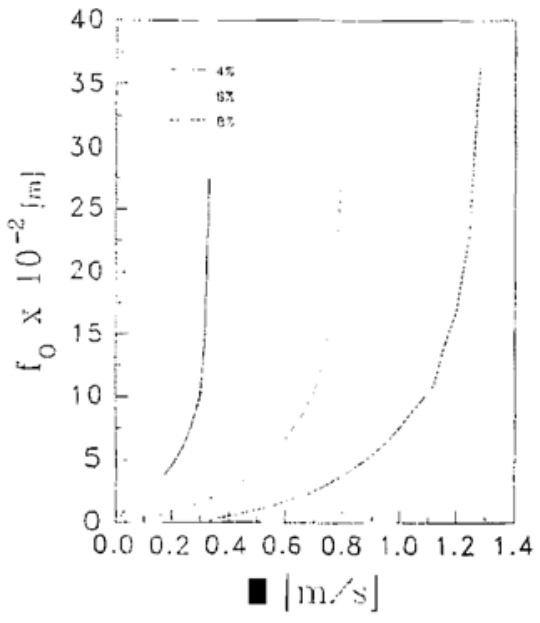

Fig. 1. Axial flame position (ncar inlet).

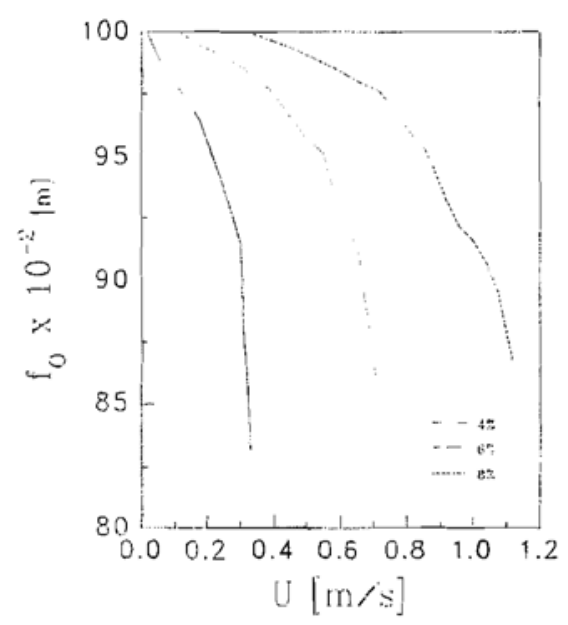

Fig. 2. Axial flame position (near outlet).

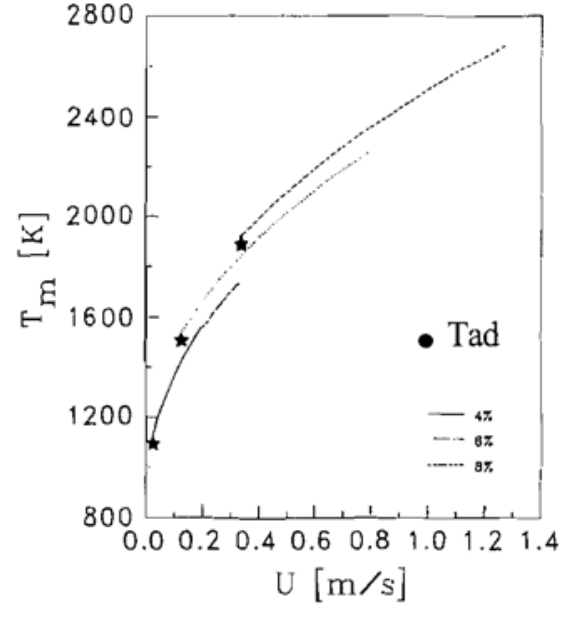

Fig. 3. Maximum temperature.

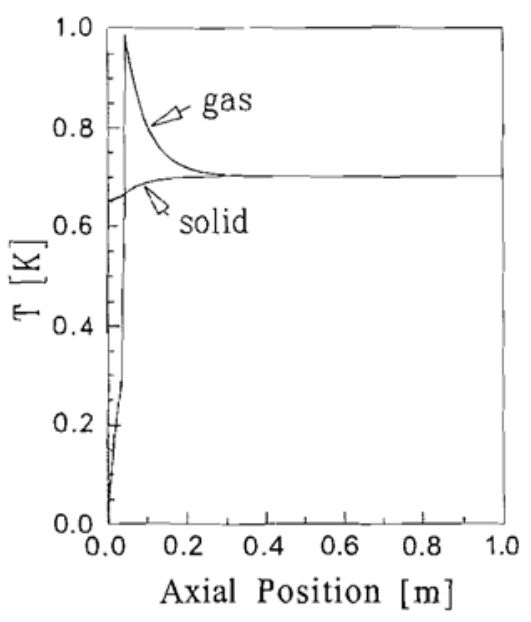

Fig. 4. Fluid and solid temperature profiles. 
Figure 4 shows the axial fluid and wall temperature profiles in the monolith for a $6 \%$ methane feed and a fluid velocity of $0.5 \mathrm{~m} / \mathrm{s}$. The overshoot is clearly seen in the fluid temperature. At the inlet of the monolith the gas temperature is lower than the solid phase temperature and the gas is preheated. The gas reaction becomes large beyond the ignition temperature and the solid-phase temperature is exceeded. In the post combustion zone, the flame temperature drops to the solid-phase temperature and the outlet temperatures (in thermal equilibrium) equal the adiabatic value.

\section{CONCLUSIONS}

The analytical solution obtained by means of matched asymptotics proves to be a very elegant method to model combustion in a porous medium. Using the same method of matched asymptotic, $\mathbf{m}$ cobedo and Vijoen (1993)found favorable comparison between the analytical solution and numerical simulation of reaction fronts in a porous medium. The analysis demonstrates clearly that the combustion temperature in monolithic structures approaches the adiabatic value at low velocities, but, as the velocity is increased, thermal overshoots occur in the gas phase. This phenomenon is more pronounced for leaner mixtures. For a mixture of $4 \%$ methane in air near the blow-off velocity, the flame temperature exceeds the adiabatic value of $1102 \mathrm{~K}$ by at least $635 \mathrm{~K}$. This represents a thermal overshoot of more than $50 \%$.

These maximum temperatures are determined with- -11t taking into consideration the endothermic dissociation reaction of $\mathrm{CO}$,. At elevated temperatures this reaction comes into play and will decrease the temperature. The actual temperature in the monolith would be less than the maximum temperature found by this analysis. Another factor which has not been included in the present analysis is heat losses at the ends of the monolith. Min and Shin (1991) did a numerical study of combustion in a monolith taking heat losses into account. The temperature of the solid phase will drop and the maximum temperature in the gas phase will also be lower. The fraction of he 1 in the feed determines the velocity range (U,, -between which a steady-state solution exits. For higher fuel feed fractions the velocity range widens and the minimum velocity increases. Density plays an important role. As the temperature of the gas phase in- creases, the velocity increases and the density de-creases. The effect of variable density is reflected by the $o$ which appears on the right-hand side of eq. (24). If it is set to one, one will obtain the result for a constant density approximation. The velocity range de- creases and the maximum gas temperature increases for the variable density case.

Acknowledgement-The authors gratefully acknowledge the financial support of the National Science Foundation through grant CTS-9308813. 


\section{NOTATION}

$\begin{array}{ll}C & \text { concentration, mol } / \mathrm{m}^{3} \\ C_{p} & \text { specific heat capacity, } \mathrm{J} / \mathrm{kg} \mathrm{K} \\ \Delta d & \text { wall thickness, m } \\ D & \text { diameter of a monolith channel, } \mathrm{m} \\ D a & \text { Damkohler number }\left[=\left(L / U_{0}\right) k_{0} e^{-\gamma}\right] \\ E & \text { activation energy, } \mathrm{J} / \mathrm{mol} \\ f & \text { axial flame position, } \mathrm{m} \\ h & \text { heat transfer coefficient, } \mathrm{W} / \mathrm{m}^{2} \mathrm{~K} \\ \Delta H & \text { heat of reaction, } \mathrm{J} / \mathrm{mol} \\ k & \text { thermal conductivity, } \mathrm{K} \\ k_{0} & \text { frequency factor, } \mathrm{m} / \mathrm{s} \\ L & \text { length of monolith, } \mathrm{m} \\ P e & \text { Peclet number }\left(=L U_{0} / \kappa\right) \\ R_{g} & \text { universal gas constant, } \mathrm{J} / \mathrm{mol} \mathrm{K} \\ t & \text { time, } \mathrm{s} \\ T & \text { temperature, } \mathrm{K} \\ U & \text { fluid velocity, } \mathrm{m} / \mathrm{s} \\ x & \text { axial position, } \mathrm{m}\end{array}$

\section{Greek letters}

$\beta \quad$ dimensionless temperature rise $[=(\mathrm{T},-$ $\left.\left.T_{\mathrm{o}}\right) ;\left(T_{m}-T_{\mathrm{o}}\right)\right]$

$\gamma \quad\left(=E / R_{g} T_{m}\right)$

$\zeta \quad$ dimensionless concentration

$\theta$ dimensionless temperature $\mathbf{\square}=\left(\mathrm{T}-T_{0}\right) /$ $\left.\left(T_{m}-T_{0}\right)\right]$

$\kappa \quad$ thermal diffusivity $\left(=k / \rho C_{p}\right)$

$\mu \quad$ dimensionless fluid velocity $\left(=U / U_{0}\right)$

$\rho$ density

$\sigma \quad\left[=T_{0} /\left(T_{m}-T_{0}\right)\right]$

$\tau \quad$ dimensionless time $\left(=U_{0} t / L\right)$

$\phi_{1} \quad$ parameter in eq. (5) $\left(=4 h_{w} L / \rho_{f} C_{p f} D U_{0}\right)$

$\phi_{2} \quad$ parameter in eq. (6) $\left(=h_{w} L / \rho_{w} C_{p_{w}} U_{0} \Delta d\right)$

$x$ dimensionless axial position $(=x / L)$

$\begin{array}{ll}\text { Subscripts } \\ 0 & \text { inlet } \\ a & \text { adiabatic } \\ \mathrm{f} & \text { fluid } \\ \mathrm{m} & \text { maximum } \\ \mathrm{I} & \text { reaction zone } \\ \mathrm{W} & \text { wall }\end{array}$

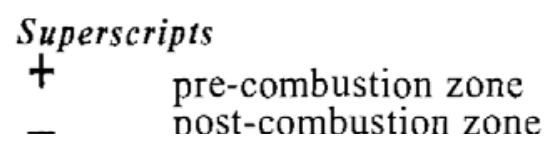




\section{REFERENCES}

Chen, T., Weng, C. and Chang, W., 1992, Transient hygrothermal stresses induced in general plane problems by theory of coupled heat and moisture $\boldsymbol{A S M E}$ J. Appl. Mech. 59, S10-S16.

Escobedo, F. A and Viljoen, H. J., 1993, Analysis of steady state reaction fronts in a porous medium. A.1.Ch.E. 3. 39, $168 \mathrm{G} 1686$.

Heck, R. H., Wei, J. and J. R.: 1976, Mathematical modeling of monolithic catalysts. A.1.Ch.E.J.22,477-484.

Matkowsky, B. J. and Sivashinsky, G. I., 1979, Acceleration effects on the stability of flame propagation. SIAM J. Appl. Math. 37,648-669.

Min, D. K. and Shin, H. D., 1991, Laminar premixed flame stabilized inside a honeycomb ceramic, J. Heat Mass Transfer 34, 341-355.

J. and Stuart, A M., 1989, A model for porous medium combustion. Q. J.Mech. Appl. Math.42,159-178.

Oh, S. H., Bissct,E. J. and Battiston, P. A., 1993, Mathematical modeling of electrically heated monolith converters: model formulation, numerical methods and experimental verification. Ind. E tgng Chern. Res. 32, 1560-1567.

Papenmeier: D. M. and Rossin, J. A, 1994, Catalytic oxidation of dichloromethane, chloroform, and their binary mixtures over a platinum alumina catalyst. Ind. Engny Chern. Res. 33, 3094-3103.

Sathe, S. B., Peck, R. E. and Tong. T. W., 1990, A numerical analysis of heat transfer and combustion in porous radiant burners. Int. J. Heat Mass Transfer 33,1331-1338,

Sih, G.C. and Ogawa, A, 1982,Transicnt thermal change on a solid surface: coupled diffusion of heat and moisture. J. Therml. Stresses 5, 265-282.

Thiart, J. J. et al., 1991, Development of thermal stresses in reacting media -I. Failure of catalyst particle. Chem. Engng Sci. 46, 351 -359. 